\title{
Alterações físicas e químicas em cebolas armazenadas sob refrigeração
}

\author{
Chemical and physical alterations in storaged onion genotypes under refrigeration
}

\author{
Cleneide Oliveira Melo ${ }^{\text {* }}$ Celso Luiz MorettiII Cristina Maria Monteiro Machado ${ }^{\mathrm{II}}$ Leonora Mansur \\ Mattos $^{\text {II }}$ Lidiane Batista Muniz ${ }^{\mathrm{I}}$
}

\section{RESUMO}

Bulbos de cebolas armazenados sob refrigeração sofrem alterações físicas e químicas. Entretanto a intensidade das alterações depende das condições de armazenamento e cultivar. Este trabalho objetivou avaliar alterações em cebolas das cultivares 'Beta Cristal' e 'Óptima' armazenadas sob refrigeração. Bulbos colhidos no campo experimental da Embrapa Hortaliças - Brasília DF, após cura, foram transportados para o laboratório onde foram selecionados $e$ armazenados a $5 \pm 1^{\circ} \mathrm{C}$ e umidade relativa de $85 \pm 5 \%$, durante 60 dias. A cada 10 dias, foram feitas análises de perda de massa, cor $\left(L^{*}\right)$, firmeza $(N)$, pungência, acidez titulável e sólidos solúveis. Observou-se, durante o armazenamento, nas duas cultivares estudadas, incremento na perda de massa, redução nos teores de sólidos solúveis e na acidez titulável. Houve grande variação na luminosidade dos bulbos durante o armazenamento. Entretanto, após 60 dias, o brilho das cebolas das duas cultivares foi significativamente maior, quando comparado ao início dos experimentos. Houve aumento significativo da firmeza dos bulbos da cultivar 'Óptima'. Ao final do experimento, a pungência foi 6 e 5 vezes maior nas cultivares 'Beta Cristal' e 'Óptima', respectivamente, em relação ao início do período de armazenamento.

Palavras-chave: cebola, qualidade, pós-colheita, temperatura.

\section{ABSTRACT}

Storage under low temperatures, immediately after the harvest, is the most used technique to extend the shelf life of different fresh produce. The present work was carried out with the objective of assessing physical and chemical alterations in onions stored under refrigeration. Onions (Allium cepa L.) cultivars 'Beta Cristal' and 'Óptima' were harvested at Embrapa Vegetables experimental fields, were cured and taken to the Food Science and Technology Lab where bulbs were selected and stored at $5 \pm 1{ }^{\circ} \mathrm{C}$ and $85 \pm 5 \%$ relative humidity for 60 days.
Every 10 days bulbs wee assessed for dry matter, mass loss (\%), brightness, firmness, titratable acidity, soluble solids and pungency. It was observed during the storage period for both cultivars an increase in weight loss, a reduction in the content of soluble solids and titratable acidity. There was a significant variation in bulbs brightness during storage. After 60 days, onions from both cultivars presented higher brightness comparing to the beginning of the experiments. There was a significant increase in firmness for 'Óptima' bulbs. At the end of the experiment, pungency was 6 and 5 times higher in 'Beta Cristal' and 'Óptima' cultivars, respectively, when compared to the values observed in the beginning of the experiment.

Key words: onion, quality, postharvest, temperature.

\section{INTRODUÇÃO}

A cebola (Allium cepa L.) é uma das mais importantes hortaliças cultivadas no mundo. É valorizada por seu característico sabor picante e constitui um dos principais condimentos utilizados para realçar o sabor dos alimentos (LANZOTTI, 2006).

O consumo de cebolas processadas aumentou nas últimas décadas. Contudo, a industrialização de cebola ainda é pouco praticada no Brasil, sendo que há pequena produção de cebola desidratada, na forma de pó ou de flocos e como picles. (FILGUEIRA, 2000). Entretanto, segundo MORETTI \& DURIGAN (2002), o mercado para produtos à base de cebola processada tem experimentado crescimento significativo.

Uma das características mais importantes da cebola para a industrialização é o seu teor de sólidos

\footnotetext{
IDepartamento de Nutrição, Faculdade de Ciências da Saúde, Campus Universitário Darcy Ribeiro, 70910-900, Brasília, DF, Brasil. E-mail: coliveiramelo@gmail.com.*Autor para correspondência.

${ }^{\mathrm{II}}$ Embrapa Hortaliças, Gama, DF, Brasil.
} 
solúveis. Esse teor é dependente da cultivar e do clima, devendo ser o maior possível. Quanto maior o teor de sólidos solúveis, maior será a qualidade do produto final, uma vez que os açúcares, ácidos orgânicos, compostos responsáveis pela qualidade sensorial da cebola, fazem parte da composição dos sólidos solúveis (CHOPE et al., 2006)

Outro atributo de qualidade importante para cebolas destinadas ao processamento é a pungência (DHUMAL et al., 2007). Segundo SCHWIMMER \& WESTON (1961), quanto maior a concentração de ácido pirúvico, melhor a pungência dos bulbos de cebolas.

Após a colheita, os bulbos de cebola são afetados por fatores fisiológicos, bioquímicos e tecnológicos, que podem induzir perdas na qualidade do produto. Os principais fatores que induzem a deterioração dos bulbos de cebolas são a respiração, retomada do crescimento e ataque de patógenos (CHOPE et al., 2006).

O uso do armazenamento refrigerado para prolongar a qualidade pós-colheita e disponibilizar o produto durante o período entressafra permite um avanço nas possibilidades para o país se tornar um grande produtor e exportador de cebolas (MORETTI, 2004; CORTEZ et al., 2002). Entretanto, durante o armazenamento refrigerado, os bulbos da cebola são expostos às circunstâncias ambientais e atmosféricas, que podem afetar suas características fisiológicas e bioquímicas, como: alterações na composição de água, compostos responsáveis pelo sabor e aroma (UDDIN \& MACTAVISH, 2003), ácidos orgânicos (SALAMA et al., 1990), carboidratos (RUTHERFORD \& WHITTLE, 1982; BENKEBLIA et al., 2005), reguladores de crescimento e fenólicos. Porém, a intensidade das alterações depende das condições de armazenamento, tempo e cultivar (BENKEBLIA, 2000; BENKEBLIA \& VAROQUAUX, 2003).

O conhecimento a respeito da possibilidade de se utilizar cebolas armazenadas para processamento ainda é pouco divulgado na literatura. Com base no exposto, o objetivo deste estudo foi avaliar as cebolas das cv. 'Beta Cristal' e 'Óptima' quanto às características de qualidade durante o armazenamento refrigerado a $5^{\circ} \mathrm{C}$.

\section{MATERIAL E MÉTODOS}

O presente estudo foi conduzido no Laboratório de Ciência e Tecnologia de Alimentos da Embrapa Hortaliças, em Brasília-DF. Para o experimento, utilizou-se cebola (Allium cepa L.) das cultivares 'Beta Cristal' e 'Óptima'.

Após a colheita, os bulbos das cebolas Beta cristal e Óptima foram selecionados quanto a cor e peso, sem danos mecânicos e sem ocorrência de brotação, acondicionados em número de 9 em bandejas de isopor descobertas e armazenados a $5 \pm 1^{\circ} \mathrm{C}$ e umidade relativa do ar de $60 \pm 1 \%$.

$\mathrm{O}$ estudo foi conduzido em delineamento inteiramente casualizado com 14 tratamentos arranjados em esquema fatorial $2 \times 7$ ( 2 cutilvares e 7 tempos de amostragem), com 4 repetições.

A perda de massa (\%) foi por meio da relação entre a diferença das massas inicial e final dividida pela massa inicial e multiplicada por 100 .

A firmeza foi determinada no ponto mediano sobre a região equatorial de cada bulbo. Utilizou-se penetrômetro (Sammar Nordeste modelo FT327) e ponta de prova de $8 \mathrm{~mm}$. Os valores foram expressos em Newton. A cor externa foi determinada em colorímetro eletrônico (MINOLTA, Cr 200 b, Osaka, Japão). Essa medida visou a avaliar as alterações na cor dos bulbos durante o armazenamento. Utilizou-se, para cada repetição, a média das medições realizadas em 3 bulbos. As medições foram realizadas em bulbo diferentes, na parte mediana, antes e durante o período de armazenagem. Usou-se o parâmetro L para determinar a luminosidade/ brilho dos bulbos.

Os sólidos solúveis ( $\left.{ }^{\circ} \mathrm{Brix}\right)$ foram determinados por refratometria do exsudato, conforme metodologia da AOAC (1992).

A acidez titulável total foi determinada pela AOAC (1992), sendo expressa em mEq de ácido pirúvico/100g de matéria fresca.

A determinação da pungência foi baseada no método de SCHWIMMER \& WESTON (1961). Para a extração do suco, os bulbos foram cortados em cubos e triturados em mixer durante 1 minuto, usando a proporção de $10 \mathrm{~g}$ de cebola para $10 \mathrm{~mL}$ de água destilada. $\mathrm{O}$ triturado foi filtrado em papel de filtro whatmam número 4, por 10 minutos. Em seguida, $0,5 \mathrm{~mL}$ do filtrado foram transferidos para um tubo de ensaio de $40 \mathrm{~mL}$. A esse filtrado adicionou-se $1,5 \mathrm{~mL}$ de ácido tricloroacético 5\% (TCA), depois, agitou-se essa solução em vortex. A solução homogeneizada ficou em repouso por uma hora. Em seguida, adicionou-se $18 \mathrm{~mL}$ de água destilada e agitou-se novamente. Para determinação do ácido pirúvico, $1 \mathrm{~mL}$ da solução anterior foi transferido para um tubo de ensaio de $20 \mathrm{~mL}$, em seguida, adicionou-se $1 \mathrm{~mL}$ da solução de 2,4 dinitrofenilhidrazina (DNPH) e $1 \mathrm{~mL}$ de água destilada. A solução foi agitada em vortex, os tubos foram colocados em banho-maria a $37^{\circ} \mathrm{C}$ por 10 minutos e, posteriormente, colocados em água com gelo. Depois de resfriados, adicionaram-se $5 \mathrm{~mL}$ de $\mathrm{NaOH} \mathrm{a} 0,6 \mathrm{~mol} \mathrm{~L}^{-1}$ e agitou-se em vortex. A solução ficou em repouso por 5 minutos, para desenvolver a cor e, em seguida, fez-se a leitura da absorbância em espectrofotômetro (Hitachi V-1100) a 420nm. A concentração de ácido pirúvico na amostra foi calculada a partir da equação obtida por regressão linear, considerando a absorbância como 
variável dependente $(\mathrm{X})$ e a concentração (piruvato de sódio 0 a $50 \mu \mathrm{mol} \mathrm{L}^{-1}$ ) como variável independente $(\mathrm{Y})$. Os resultados foram expressos em $\mu$ mol de ácido pirúvico por grama de cebola.

Os dados foram submetidos à análise de variância e as médias foram comparadas pelo teste de diferença mínima significativa ao nível de 5\% de probabilidade.

\section{RESULTADOS E DISCUSSÃO}

Durante os 60 dias de armazenamento, observou-se perda de massa para as duas cultivares estudadas. Na 'Beta Cristal', ao final do experimento, a perda de massa foi de 3,85\%, enquanto que na 'Óptima' a perda foi de $2,50 \%$ em comparação com o início do experimento (Figura 1A).

Diversos trabalhos têm demonstrado que cebolas armazenadas sob refrigeração apresentam perda de massa, todavia essas perdas são proporcionais ao tempo e às condições de armazenamento (UDDIN \& MACTAVISH, 2003). A perda de massa em hortaliças é decorrente, principalmente, da transpiração, em resposta ao déficit de pressão de vapor (DPV) entre seus tecidos e o ambiente externo (WOODS, 1990).

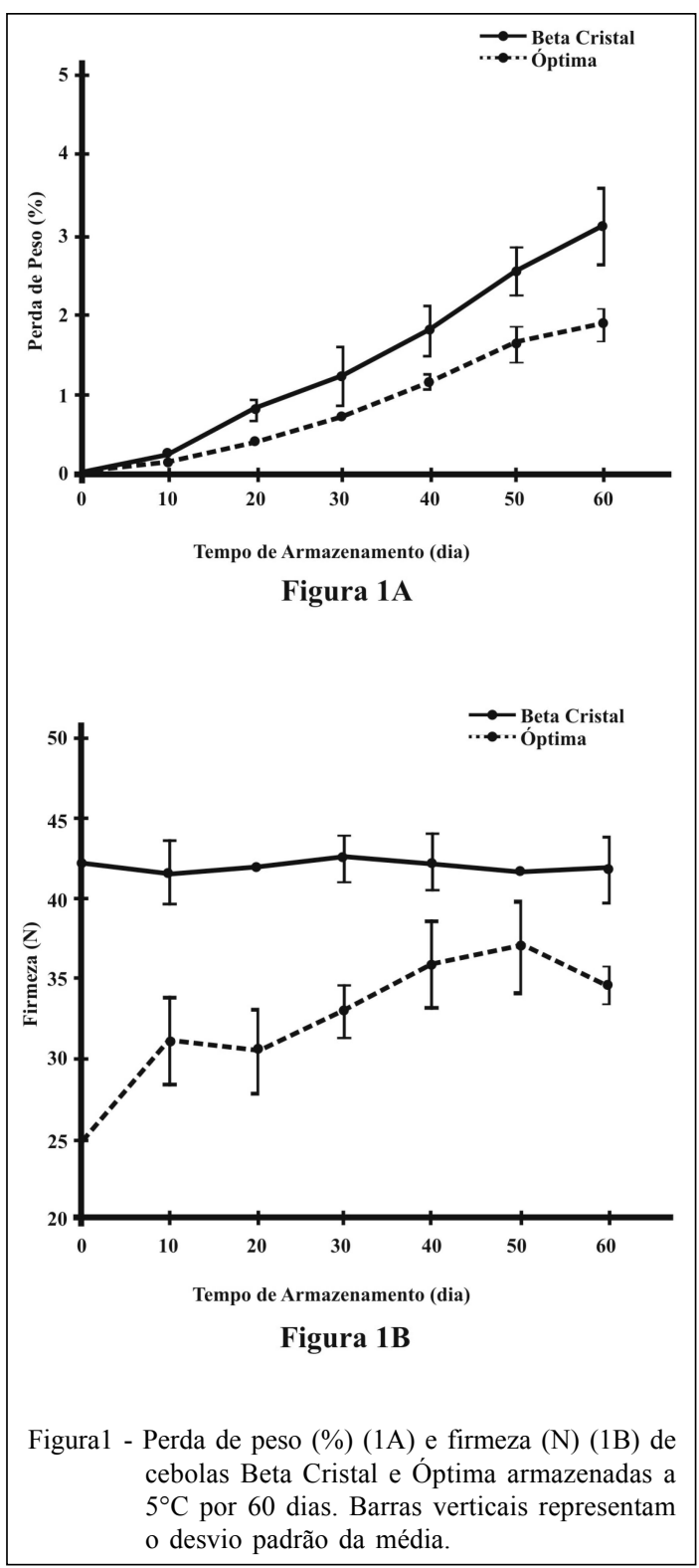

Ciência Rural, v.42, n.11, nov, 2012. 
Houve diferença significativa entre as cultivares avaliadas para firmeza. Ao final de 60 dias de armazenamento, observou-se que a firmeza dos bulbos da cultivar 'Beta Cristal' manteve-se constante, enquanto que na cultivar 'Óptima' a firmeza aumentou (Figura 1B).

Os resultados encontrados para as cultivares estudadas não foram concordantes aos encontrados por CHOPE et al. (2006). Em seu estudo, o autor constatou diminuição da firmeza em cebolas durante armazenamento refrigerado. A diminuição da firmeza em frutas e hortaliças está associada a reações bioquímicas de degradação das paredes celulares, durante processo de amadurecimento, senescência e deterioração, entretanto, há variabilidade de conservabilidade entre as cultivares (MORETTI et al., 2000; KHOKHAR, 2009).

Durante os 60 dias de armazenamento, observou-se que houve aumento dos valores de luminosidade para as cultivares 'Beta Cristal' e 'Óptima' (Figura 2A). A partir desses resultados, entendeu-se que, ao final do armazenamento, os bulbos das cultivares 'Beta Cristal' e 'Óptima' estavam mais claros, uma vez que o parâmetro L varia de 0 a 100, sendo o valor 0 indicativo da cor preta e o valor 100, do branco (MCGUIRE, 1992; SHEWFELT, 1999). Ao final do experimento, verificou-se

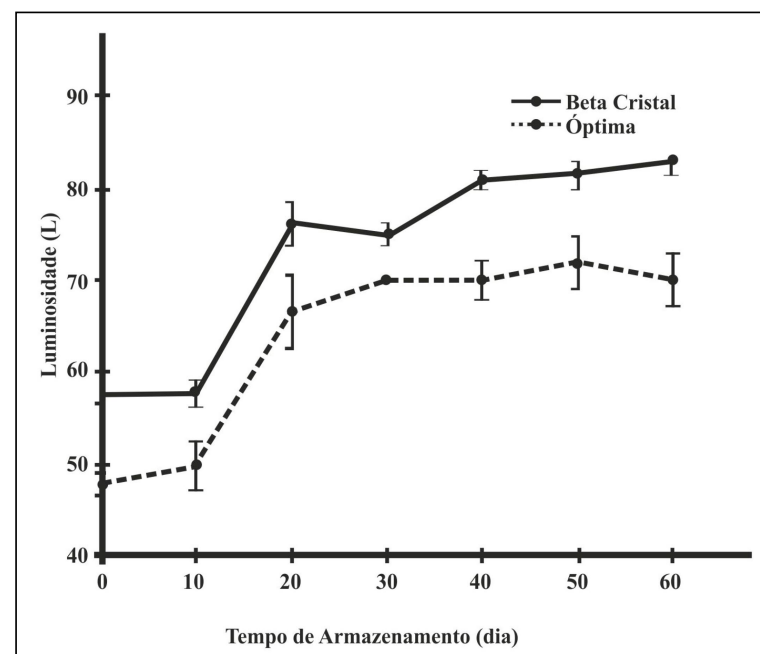

Figura 2A

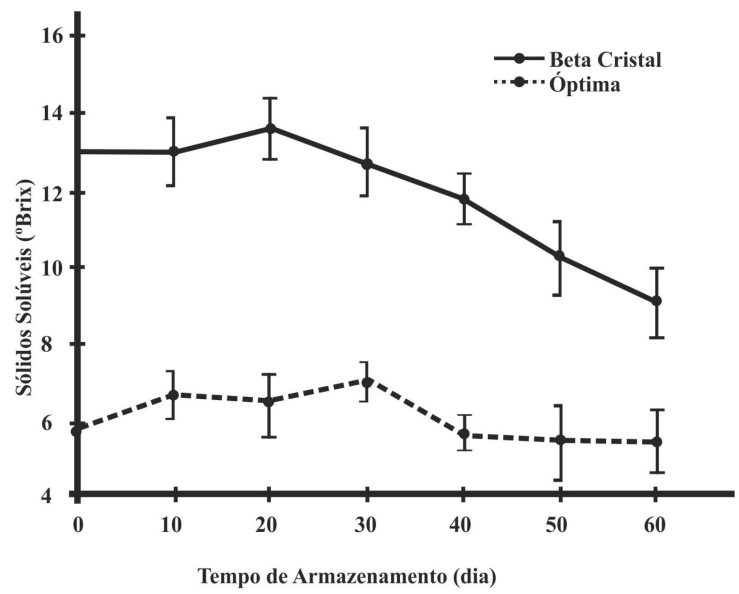

Figura 2B

Figura 2 - Luminosidade (2A) e sólidos solúveis ( ${ }^{\circ}$ Brix) (2B) de cebolas Beta Cristal e Óptima, armazenadas a $5^{\circ} \mathrm{C}$ por 60 dias. Barras verticais representam o desvio padrão da média.

Ciência Rural, v.42, n.11, nov, 2012. 
que a luminosidade da cultivar 'Beta Cristal' era $15 \%$ maior que o da cultivar 'Óptima'.

Nas duas cultivares avaliadas, os teores de sólidos solúveis diminuíram de maneira distinta durante os 60 dias de armazenamento refrigerado (Figura 2B), sendo que tal evento foi mais pronunciado na cultivar 'Beta Cristal'. CHOPE et al. (2006), em seu estudo, relataram um pico na concentração dos sólidos solúveis entre 4 e 6 semanas de armazenamento nas cultivares 'Renate', 'SS1' e 'Ailsa Craig', seguidos por diminuição média de $18 \%$ até 70 dias de armazenamento. Por outro lado, ABRAMETO et al. (2010) não encontraram alteração significativa na concentração de sólidos solúveis entre o início e o fim do armazenamento refrigerado em cebolas Valuno e Valcatorce, similarmente ao verificado para a cultivar 'Óptima'.

As reduções dos teores de sólidos solúveis verificadas para as cultivares estudadas provavelmente estão associadas com o consumo dos carboidratos durante a respiração (RUTHERFORD \& WHITTLE, 1982; CHOPE et al., 2006).

A acidez mais elevada foi encontrada na cultivar 'Beta Cristal', no entanto, durante o armazenamento, a acidez não apresentou alteração significativa, quando comparada ao início do armazenamento (Figura 3A). Na cultivar 'Óptima', a

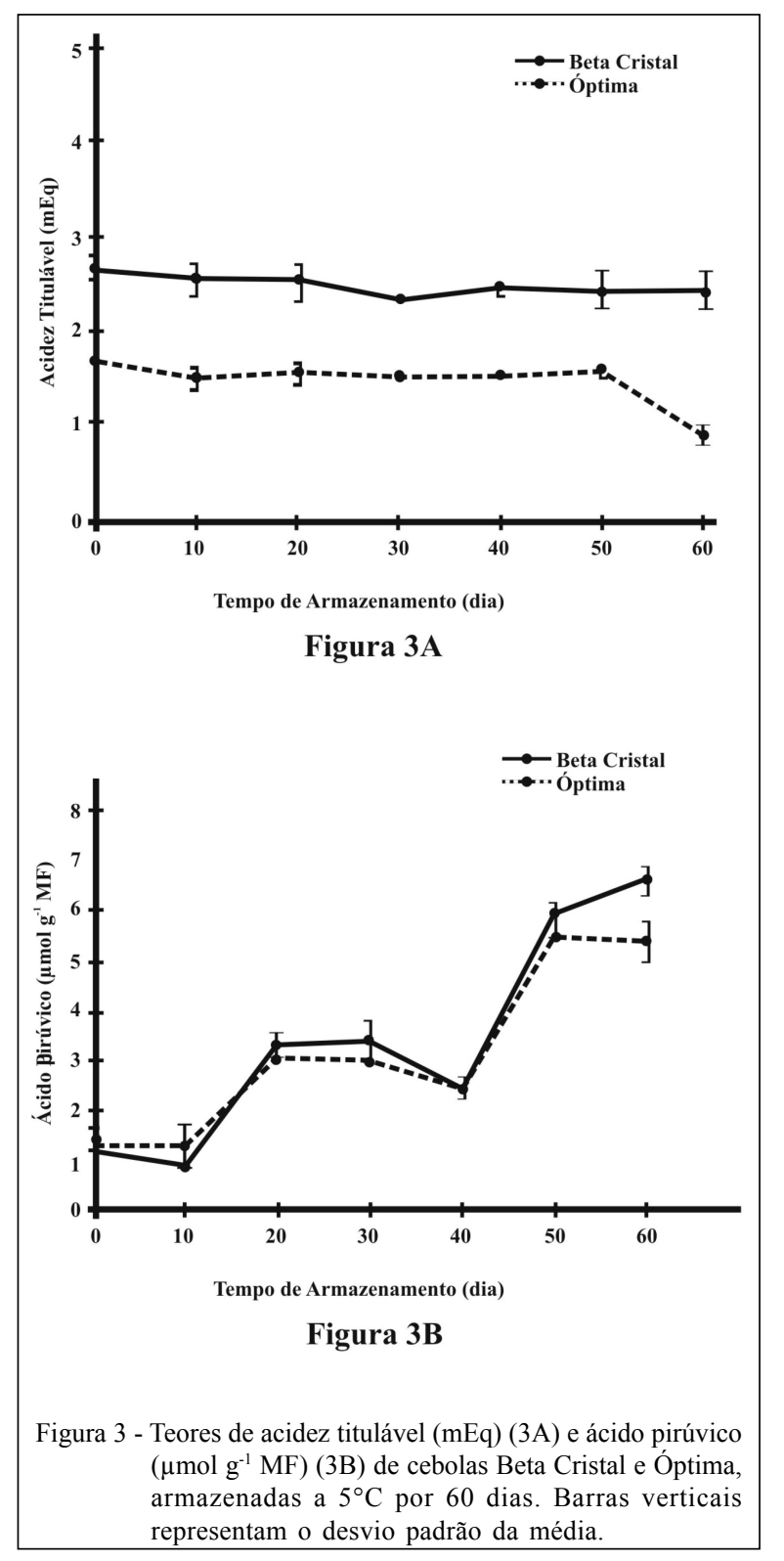

Ciência Rural, v.42, n.11, nov, 2012. 
acidez manteve-se constante durante o armazenamento. A partir de tempo de 50 dias até o final do armazenamento, observou-se uma redução significativa.

É desejável que o bulbo a ser processado contenha um alto teor de pungência, por haver perda dessa característica durante o processamento (CHITARRA\& CHITARRA, 2005).

Verificou-se tendência similar para a pungência nas duas cultivares estudadas durante o armazenamento (Figura 3B). Após 10 dias, houve um

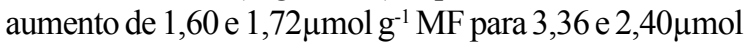
$\mathrm{g}^{-1}$ MF nas cultivares 'Beta Cristal' e 'Óptima', respectivamente, seguido por uma ligeira diminuição até 40 dias de armazenamento. Após 50 dias de armazenamento, os valores aumentaram significativamente, sendo que, ao final do experimento, a concentração de ácido pirúvico era 6 vezes maior para a cultivar 'Beta Cristal' e 5 para a 'Óptima'.

$O$ aumento da pungência durante o armazenamento concorda com as observações de BENKLEBIA (2000). Para a cultivar 'Rouge Amposta', armazenada a $4^{\circ} \mathrm{C}$, o autor encontrou um aumento de $10 \mu \mathrm{mol} \mathrm{g} \mathrm{g}^{-1} \mathrm{MF}$ após 10 semanas de armazenamento. CHOPE et al. (2006) verificaram aumento do ácido pirúvico nas cultivares 'Renate' e 'Alisa Craig' de 1,9 e 1,2 vezes, respectivamente, durante o período de armazenamento refrigerado.

A pungência da cebola é produzida pela hidrólise de uma substância precursora, sulfóxido de $S$ alk(en)il-L- cisteína, quando a célula sofre ruptura mecânica. Essa reação produz o ácido pirúvico e outros compostos sulfurados (MACTAVISH, 2003; CHOPE et al., 2006). SCHWIRMMER \& WESTON (1961) classificaram a pungência medida em função da quantidade de ácido pirúvico, como cebola fraca $(2 \mathrm{a} 4 \mu \mathrm{mol}$ $\left.\mathrm{g}^{-1}\right)$, intermediária $\left(8 \mathrm{a} 10 \mu \mathrm{mol} \mathrm{g}^{-1}\right)$ e forte $\left(15 \mathrm{a} 20 \mu \mathrm{mol} \mathrm{g}^{-1}\right)$.

\section{CONCLUSÃO}

As cebolas das cultivares 'Beta Cristal' e 'Óptima armazenadas a $5^{\circ} \mathrm{C}$ por 60 dias apresentaram incremento na perda de massa, luminosidade e teores de ácido pirúvico, além de ligeira redução nos teores de sólidos solúveis e acidez titulável, porém sem comprometimento da qualidade para o processamento.

\section{REFERÊNCIAS}

ASSOCIATION OF OFFICIAL ANALYTICAL CHEMIST (AOAC). Official methods of analysis of the Association of Official Analytical Chemistry. Washigton, 1992. 115p.

ABRAMETO, MA. et al. Analysis of methodologies for the study of composition and biochemical carbohydrate changes in harvest and postharvest onion bulbs. International Journal of Experimental Botany, v.79, p.123-132, 2010. Disponível em: <http://revistaphyton.fund-romuloraggio.org.ar/vol179/ Abrameto.pdf $>$. Acesso em: 02 fev. 2011.

BENKEBLIA, N. Phenylalanine ammonia-lyase, peroxidase, pyruvic acid and total phenolics variations in onion bulbs during long-term storage. Food Science and TechnologyLebensmittel-Wissenschaft \& Technologie, v.33, p.112116, 2000. Disponível em: <linkinghub.elsevier.com/retrieve/ pii/S0308814698001563>. Acesso em: 07 mar. 2011. doi: $10.1006 /$ fstl.1999.0624.

BENKEBLIA, N.; VAROQUAUX, P. Effect of nitrous oxide $\left(\mathrm{N}_{2} \mathrm{O}\right)$ on respiration rate, soluble sugars and quality attributes of onion bulbs Allium cepa cv. 'Rouge Amposta' during storage. Postharvest Biology and Technology, v.30, p.161-168, 2003. Disponível em: <linkinghub.elsevier.com/retrieve/pii/ S0925521403001017>. Acesso em: 07 mar. 2011. doi: $10.1016 / \mathrm{S} 0925-5214(03) 00101-7$.

BENKEBLIA, N. et al. Variation in 1-fructoexohydrolase (1FEH) and 1-kestose-hydrolysing (1-KH) activities and fructooligosaccharide (FOS) status in onion bulbs. Influence of temperature and storage time. Journal of the Science of Food and Agriculture, v.85, p. 27-234, 2005. Disponível em: $<$ linkinghub.elsevier.com/retrieve/pii/S0308814603006356> Acesso em: 07 mar. 2011. doi: 10.1016/j.foodchem.2003.12.010.

CHITARRA, M.I.F.; CHITARRA, A.B. Pós-colheita de frutos e hortaliças: fisiologia e manuseio. Lavras: ESALQ/FAEP, 2005. 783p.

CHOPE, G.A. et al. Effect of controlled atmosphere storage on abscisic acid concentration and other biochemical attributes of onion bulbs. Postharvest Biology and Technology, v.39. p.233-242, 2006. Disponível em: <linkinghub.elsevier.com/ retrieve/pii/S0925521405002218>. Acesso em: 10 mar. 2011. doi: $10.1016 /$ j.postharvbio.2005.10.010.

CORTEZ, L.A.B. et al. Resfriamento de frutas e hortaliças Brasília, DF: Embrapa Hortaliças, Embrapa Informações Tecnológica, 2002. 428p.

DHUMAL, K. et al. Assessment of bulb pungency level different indian cultivars of onion (Allium cepa L.). Food Chemistry, v.100, p.1328-1330, 2007. Disponível em: $<\mathrm{http}: /$ www.sciencedirect.com/ science/article/pii/S0308814605010514>. Acesso em: 25 mar. 2011. doi: 10.1016/j.foofchem.2005.11.044

FILGUEIRA, F.A.R. Novo manual de olericultura agrotecnologia moderna na produção e comercialização de hortaliças. Viçosa: UFV, 2000. 402p.

KHOKHAR, K.M. Effect of set-size and storage temperature on bolting, bulbing and seed yield in two onion cultivars. Scientia Horticulturae, v.122, p.187-194, 2009. Disponível em: <http:// www.sciencedirect.com/science/article/pii/S030442380900257X>. Acesso em: 05 maio, 2011. doi: 10.16/ j .scienta.2009.05.008.

LANZOTTI, V. The analysis of onion and garlic. Journal of Chromatography A, v.1112, p.3-22, 2006. Disponível em: $<\mathrm{http}$ :/ /www.sciencedirect.com/science/article/pii/S0021967305024064>. Acesso em: 20 maio, 2011. doi: 10.1016/j.croma.2005.12016.

MACTAVISH, U.M.H.S. Controlled atmosphere and regular storage-induced changes in $S$-alk(en)yl-1-cysteine sulfoxides and 
alliinase activity in onion bulbs (Allium cepa L. cv. 'Hysam'). Postharvest Biology and Technology, v.28, p.239-245, 2003. Disponível em: <linkinghub.elsevier.com/retrieve/pii/ S0925521402001953>. Acesso em: 10 mar. 2011. doi: $10.1016 / \mathrm{S} 0925-5214(02) 00195-3$.

MCGUIRE, R.G. Reporting of objective color measurements. Hort Science, v.27, p.1254-1255, 1992.

MORETTI, C.L. Colheita e manuseio pós-colheita. Sistema de produção de cebola (Allium cepa L), 2004. Disponível em: <http://www.cnph.embrapa.br/sistprod/cebola/ colheita_e_pos.htm>. Acesso em: 20 mar. 2011

MORETTI, C.L. et al. Metabolismo respiratório na pós-colheita de frutas e hortaliças. Universa, v.8, n.1, p.259-273, 2000.

MORETTI, C.L.; DURIGAN, F.J. Processamento de cebola. Informe Agropecuário, v.23, p.99-104, 2002.

RUTHERFORD, R.; WHITTLE, R. The carbohydrate composition of onions during long term cold storage. The
Journal of horticultural Science \& Biotechnology, v.57, p.249-356, 1982 .

SALAMA, A.M. et al. Sugar and organic acid changes in stored onion bulbs treated with maleic hydrazide. HortScience, v.25, p.1625-1628, 1990.

SCHWIMMER, S.; WESTON W.J. Enzimatic development of pyruvic acid as a measure of pungency. Journal Agricultural Food Chemistry, v.9, p.301-304, 1961.

SHEWFELT, R.L. What is quality? Postharvest Biology and Technology, v.15, p.197-200, 1999.

UDDIN, M.M.; MACTAVISH, H.S. Controlled atmosphere and regular storage-induced changes in S-alk(en)yl-L-cysteine sulfoxides and alliinase activity in onion bulbs (Allium cepa L. cv. 'Hysam'). Postharvest Biology and technology, v.28, p.239-245, 2003. Disponível em: <linkinghub.elsevier.com/ retrieve/pii/S0925521406003486>. Acesso em: 18 mar. 2011.

WOODS, J.L. Moisture loss from fruits and vegetables. Postharvest News and Information, v.1, n.3, p.195-199, 1990. 